\title{
An Existence Theorem for Multimeron Solutions to Classical Yang-Mills Field Equations ${ }^{\star}$
}

\author{
T. Jonsson ${ }^{1 \star \star}$, O. McBryan ${ }^{1 \star \star \star}$, F. Zirilli ${ }^{1+}$, and J. Hubbard ${ }^{2++}$ \\ 1 Harvard University, Cambridge, Massachusetts 02138, USA \\ 2 Cornell University, Ithaca, New York 14853, USA
}

\begin{abstract}
In this paper we prove the existence of solutions to a class of boundary value problems for a singular nonlinear elliptic partial differential equation in a half plane. By a recent paper of J. Glimm and A. Jaffe, this proves the existence of multimeron solutions to the classical SU(2) Yang-Mills field equations in Euclidean space.
\end{abstract}

\section{Introduction and Results}

In this paper we prove existence of solutions to a class of boundary value problems for the singular elliptic equation

$$
r^{2} \Delta \psi=\psi^{3}-\psi
$$

in the half plane $\mathbb{R}_{+}^{2}=\left\{(r, t) \in \mathbb{R}^{2} \mid r>0\right\}$, where $\Delta=\frac{\partial^{2}}{\partial r^{2}}+\frac{\partial^{2}}{\partial t^{2}}$. We remark that $r^{2} \Delta$ is the Laplace-Beltrami operator for the Poincare halfplane $\left[\mathbb{R}_{+}^{2}\right.$ with the metric $\left.r^{-2}\left(d r^{2}+d t^{2}\right)\right]$. The boundary values are specified by an increasing sequence $\left\{t_{i} \mid 1 \leqq i \leqq 2 n\right\}$ of real numbers and the requirement

$$
\begin{aligned}
& \lim _{r \rightarrow 0} \psi(r, t)=(-1)^{i}, \quad t_{i}<t<t_{i+1}, \quad i=0, \ldots, 2 n, \\
& \lim _{(r, t) \rightarrow \infty} \psi(r, t)=1,
\end{aligned}
$$

where we have defined $t_{0}=-\infty, t_{2 n+1}=\infty$. Our principal result is

Theorem 1.1. There is a function $\Psi$, real analytic and satisfying (1.1) in $\mathbb{R}_{+}^{2}$, which assumes the boundary values (1.2).

$\star$ Supported in part by the National Science Foundation under Grant PHY 77-18762

$\star \star$ Supported in part by the Icelandic Science Foundation

$\star \star \star$ Permanent address : Department of Mathematics, Cornell University, Supported in part by Grant DMR 77-04105

+ Permanent address: Istituto Matematico G. Castelnuovo, Università di Roma, I-Rome, Italy

++ Supported in part by Grant MCS 76-06524 
In the past few years considerable progress has been made towards the understanding of classical gauge field theories, particularly for an SU(2) gauge group in four dimensional Euclidean space. Various explicit solutions have been found - the instantons, see e.g. [1-3], and the two-meron solution of DeAlfaro et al. [4]. Instantons are characterized by having finite action and integer-valued topological charge, while merons have topological charge density $\pm \frac{1}{2}$ concentrated at points, which leads to infinite action. In recent papers of Callan et al. [5] and of Glimm and Jaffe [6], it is argued that merons might provide a mechanism for quark confinement in non-abelian gauge quantum field theories. Glimm and Jaffe [7] reduced the problem of finding cylindrically symmetric multiple-meron solutions of the SU(2) Yang-Mills equations to the solution of the above boundary value problem. The points $\left\{\left(0, t_{i}\right)\right\}$ are interpreted as the location of the merons, the $t$-axis is the axis of cylindrical symmetry and the $r$-coordinate is the distance from this axis. Thus Theorem 1.1 proves existence of multiple-meron solutions to the Yang-Mills equations with merons located on a straight line.

Formally (1.1) is the Euler-Lagrange equation of the functional

$$
A(\psi)=\int_{\mathbb{R}_{+}^{2}} d^{2} x\left\{\frac{1}{2}(\nabla \psi)^{2}+\frac{1}{4} r^{-2}\left(\psi^{2}-1\right)^{2}\right\} .
$$

However, it is not hard to show that $A(\psi)$ is infinite for $\psi$ which satisfy the boundary conditions. In order to employ variational methods to solve (1.1) we define a renormalized action (energy) functional $A_{R}$. Let $\Psi_{0}$ be a $C^{x_{2}}$ function on $\mathbb{R}_{+}^{2}$ satisfying the boundary conditions (1.2). Then $\psi=\Psi_{0}+\varphi$ is a solution of (1.1) and (1.2) if and only if

$$
\Delta \phi=f+r^{-2}\left\{\left(3 \Psi_{0}^{2}-1\right) \phi+3 \Psi_{0} \phi^{2}+\phi^{3}\right\}
$$

and

$$
\lim _{r \rightarrow 0} \phi(r, t)=0=\lim _{(r, t) \rightarrow \infty} \phi(r, t),
$$

where

$$
f=-\Delta \Psi_{0}+r^{-2}\left(\Psi_{0}^{3}-\Psi_{0}\right) .
$$

We observe that (1.4) is obtained by varying the functional

$$
A_{R}(\phi)=\int_{\mathbb{R}_{+}^{2}} d^{2} x\left[\frac{1}{2}(\nabla \phi)^{2}+f \phi+r^{-2}\left\{\frac{3 \Psi_{0}^{2}-1}{2} \phi^{2}+\Psi_{0} \phi^{3}+\frac{1}{4} \phi^{4}\right\}\right] \text {. }
$$

Let $\left\{M_{n}\right\}_{n=1}^{\infty}$ be a family of bounded open subsets of $\mathbb{R}_{+}^{2}$ with the following properties:
i) $M_{n} \subset M_{n+1}$.
ii) $\inf \left\{r \mid(r, t) \in M_{n}\right\}>0$.
iii) $\partial M_{n}$ is smooth.
iv) $\bigcup_{n=1}^{\infty} M_{n}=\mathbb{R}_{+}^{2}$.

For $\Omega \cong \mathbb{R}_{+}^{2}$ define

$$
A_{\Omega}(\psi)=\int_{\Omega} d^{2} x\left\{\frac{1}{2}(\nabla \psi)^{2}+\frac{1}{4} r^{-2}\left(\psi^{2}-1\right)^{2}\right\} .
$$

Then

$$
A_{R}(\phi)=\lim _{n \rightarrow \infty}\left[\mathrm{A}_{M_{n}}\left(\Psi_{0}+\phi\right)-\mathrm{A}_{M_{n}}\left(\Psi_{0}\right)\right] .
$$


This justifies the name renormalized functional. Throughout the paper we consider a fixed family $\left\{M_{n}\right\}_{n=1}^{\infty}$ as above.

While mathematicians generally study finite action solutions to elliptic equations, definiton (1.7) reduces the study of certain infinite action solutions of (1.1) to the study of finite action solutions of (1.4). This trick, isolating the infinite part of the action, is the mathematics of what physicists call vacuum energy renormalization.

Our solution to (1.1) and (1.2) is obtained by choosing an approximate solution, $\Psi_{0}$ as above and then solving (1.4) by minimizing $A_{R}$ on a family of Hilbert spaces. At the end we extract a solution to (1.4) and (1.5) by a compactness argument. As an approximate solution we take

$$
\Psi_{0}(r, t)=\cos \left\{\sum_{i=1}^{2 n}(-1)^{i} \arctan \frac{t-t_{i}}{r}\right\}
$$

which satisfies the boundary conditions (1.2). It is clear that the corresponding $f$ is real analytic and in the Appendix we show that $f$ is bounded and $O\left(\left(r^{2}+t^{2}\right)^{-2}\right)$ at infinity.

Let $\Omega \subset \mathbb{R}_{+}^{2}$ be open and denote by $C^{\infty}(\Omega)$ the set of all $C^{\infty}$ functions on $\mathbb{R}_{+}^{2}$ restricted to $\Omega$. Let $C_{0}^{\infty}(\Omega)$ be the collection of those functions in $C^{\infty}(\Omega)$ that have compact supports contained in $\Omega$. We use the notation $\|\cdot\|_{p},\|\cdot\|_{p, \mu}$ for the $L^{p}$-norms with respect to the measures $d^{2} x$ and $d \mu=r^{-2} d^{2} x$, respectively $\left(d^{2} x\right.$ is Lebesgue measure), while $\|\cdot\|_{p, \Omega},\|\cdot\|_{p, \mu, \Omega}$ denote the corresponding norms with $d^{2} x$ replaced by $\chi_{\Omega} d^{2} x$. Here $\chi_{\Omega}$ is the characteristic function of a measurable set $\Omega \subset \mathbb{R}_{+}^{2}$.

Let $W^{p, q}(\Omega)$ denote the completion of $C^{\infty}(\Omega)$ in the norm

$$
\|\phi\|_{p, q}=\sum_{\substack{\alpha+\beta \leq p \\ \alpha, \beta \in \overline{\mathbb{Z}}^{0}}}\left\|\frac{\partial^{\alpha+\beta}}{\partial t^{\alpha} \partial r^{\beta}} \phi\right\|_{q, \Omega}, \quad p \in \mathbb{Z}^{+} .
$$

The completion of $C_{0}^{\infty}(\Omega)$ in the same norm is denoted $W_{0}^{p, q}(\Omega)$. To simplify our notation we write $B_{\Omega}$ for $W_{0}^{1,2}(\Omega)$ and $B_{n}$ for $B_{M_{n}}$. We note that $B_{\Omega}$ is a separable Hilbert space and there is a natural isometric imbedding of $B_{\Omega}$ into $B_{\Omega^{\prime}}$ if $\Omega \subset \Omega^{\prime}$. In the sequel we use this imbedding without explicit notation.

Let $\Omega \subset \mathbb{R}_{+}^{2}$ be bounded and define $\|\phi\|_{B}=\|\nabla \phi\|_{2, \Omega}$ for $\phi \in B_{\Omega}$. The norms $\|\cdot\|_{B}$ and $\|\cdot\|_{1.2}$ are equivalent on $B_{\Omega}$ (see e.g. [9]). We denote by $B$ the completion of $C_{0}^{\infty}\left(\mathbb{R}_{+}^{2}\right)$ in the norm $\|\nabla(\cdot)\|_{2}$. Then $B_{\Omega} \subset B$ for $\Omega$ bounded.

Our main technical tools for proving Theorem 1.1 are the following two estimates:

E1. There are positive constants $C_{1}$ and $C_{2}$ such that for all $\phi \in B$

$$
A_{R}(\phi) \geqq C_{1}\|\phi\|_{B}^{2}-C_{2} .
$$

E 2. If $\phi \in B$ is a smooth solution to (1.4), then

$$
|r \nabla \phi| \leqq \text { constant }
$$

We show in the appendix that $A_{R}$ is strongly continuous on $B_{\Omega}$ provided $\bar{\Omega}$ is compact in $\mathbb{R}_{+}^{2}$. In Sect. II we prove that $A_{R}$ is weakly lower semicontinuous on $B_{\Omega}$ 
under the same hypothesis. In Sect. II we also prove a stronger version of E1. The estimate E1 and the weak lower semicontinuity imply that there is $\phi_{\Omega} \in B_{\Omega}$ at which $A_{R}$ attains its infimum on $B_{\Omega}$. It follows that $\phi_{\Omega}$ is a weak solution of (1.4) in $\Omega$. Letting $\left\{M_{n}\right\}_{n=1}^{\infty}$ be as above, the sequence $\left\{\phi_{M_{n}}\right\}_{n=1}^{\infty}$ is bounded in $B$ and therefore has a weakly convergent subsequence with limit $\Phi$ say. The function $\Phi$ is a weak solution of (1.4) in $\mathbb{R}_{+}^{2}$.

In Sect. III we show that $\Phi$ is real analytic in $\mathbb{R}_{+}^{2}$ and $\Phi \rightarrow 0$ at the boundary of $\mathbb{R}_{+}^{2}$. To prove the second of these statements we use E2.

\section{Existence of Weak Solutions}

We begin by recalling some results of functional analysis. For a detailed exposition of the techniques used here see e.g. Vainberg [8]. In the following, $X$ will denote a separable reflexive Banach space with norm $\|\cdot\|$.

Proposition 2.1. Let $G$ be a weakly lower semi-continuous real-valued functional on $X$ such that $G(u) \rightarrow \infty$ as $\|u\| \rightarrow \infty$. Then $G(u)$ is bounded below and attains its infimum on $X$.

For a proof see $[8$, p. 93].

We wish to apply Proposition 2.1 to the functional $A_{R}$ on the Hilbert spaces $B_{n}$ of Sect. I. The weak lower semicontinuity of $A_{R}$ on $B_{n}$ is proven in Theorem 2.3. Theorem 2.7, which implies estimate E1, establishes that $A_{R}(\phi) \rightarrow \infty$ as $\|\phi\|_{B} \rightarrow \infty$. Thus, Proposition 2.1 and Theorems 2.3 and 2.7 prove the existence of $\phi_{n} \in B_{n}$ minimizing $A_{R}$ on $B_{n}$.

Proposition 2.2. A strongly continuous convex functional on $X$ is weakly lower semicontinuous.

For a proof see $[8$, p. 87$]$.

Theorem 2.3. Let $\Omega \subset \mathbb{R}_{+}^{2}$ be open and $\bar{\Omega}$ compact in $\mathbb{R}_{+}^{2}$. Then $A_{R}$ is weakly lower semi-continuous on $B_{\Omega}$.

Proof. For $\phi \in B_{\Omega}$ we have

$$
A_{R}(\phi)=\int_{\Omega}\left\{\frac{1}{2}(\nabla \phi)^{2}+f \phi+r^{-2}\left[\frac{3}{2} \Psi_{0}^{2} \phi^{2}+\Psi_{0} \phi^{3}+\frac{1}{4} \phi^{4}\right]\right\} d^{2} x-\frac{1}{2} \int_{\Omega} r^{-2} \Psi_{0}^{2} \phi^{2} d^{2} x .
$$

The first integral above is a convex function of $\phi$. It is also strongly continuous since $f, r^{-2}, \Psi_{0}$ are bounded functions on $\Omega$ and $\Omega$ is bounded, cf. Appendix. By Proposition 2.2 we conclude that the first integral is weakly lower semicontinuous. The second integral is a weakly continuous function of $\phi$, since the imbedding

$$
B_{\Omega} \rightarrow L^{2}(\Omega)
$$

is compact (see e.g. [9]) and $r^{-2} \Psi_{0}^{2}$ is bounded on $\Omega$.

Before proving Theorem 2.7 and the estimate E1, we establish three inequalities. 
Lemma 2.4. If $u \in C_{0}^{\infty}((0, \infty))$, then $\int_{0}^{\infty} d r\left(\frac{d u}{d r}\right)^{2} \geqq \frac{1}{4} \int_{0}^{\infty} d r \frac{u^{2}}{r^{2}}$.

Proof. Integrate $u^{2} / r^{2}$ by parts and use the Schwarz inequality.

Lemma 2.5. If $u \in C_{0}^{\infty}((-\pi / 2, \pi / 2))$, then for $a \in[0,1]$ :

$$
\int_{-\pi / 2}^{\pi / 2} d \alpha\left(\frac{d u}{d \alpha}\right)^{2} \geqq \int_{-\pi / 2}^{\pi / 2} d \alpha\left\{a^{2} u^{2}+\left(a-a^{2}\right) u^{2} \sec ^{2} \alpha\right\}
$$

Proof.

$$
\int_{-\pi / 2}^{\pi / 2} d \alpha\left(u^{2} \sec ^{2} \alpha\right)=-2 \int_{-\pi / 2}^{\pi / 2} d \alpha\left[\frac{d u}{d \alpha} u \tan \alpha\right] \leqq \int_{-\pi / 2}^{\pi / 2} d \alpha\left\{a^{-1}\left(\frac{d u}{d \alpha}\right)^{2}+a u^{2} \tan ^{2} \alpha\right\} .
$$

The lemma follows immediately since $\sec ^{2} \alpha=1+\tan ^{2} \alpha$.

Definition. For $\lambda>0, \varepsilon>0$ we define $D_{\varepsilon, \lambda}$ on $C_{0}^{\infty}((-\pi / 2, \pi / 2))$ by

$$
D_{\varepsilon, \lambda}(u)=\int_{-\pi / 2}^{\pi / 2} d \alpha\left[\left(\frac{d u}{d \alpha}\right)^{2}-(1+\varepsilon) u^{2}+\left(\lambda \sin \alpha+(2 \lambda)^{-1} u\right)^{2} u^{2}\right] .
$$

Lemma 2.6. There is an $\varepsilon_{0} \in[0,3 / 4]$ such that for $u \in C_{0}^{\infty}((-\pi / 2, \pi / 2))$ and $\lambda \in[1 / 2,3 / 4]$

$$
D_{\varepsilon_{0}, \lambda}(u) \geqq 0 \text {. }
$$

Proof. We write $u=u_{+}+u_{-}$where $u_{+}$and $u_{-}$are even and odd, respectively under the transformation $\alpha \rightarrow-\alpha$. Since the inner product in $L^{2}((-\pi / 2, \pi / 2))$ between an even and an odd function vanishes,

$$
\begin{aligned}
D_{\varepsilon, \lambda}(u)= & \int_{-\pi / 2}^{\pi / 2} d \alpha\left[\left(\frac{d u_{+}}{d \alpha}\right)^{2}+\left(\frac{d u_{-}}{d \alpha}\right)^{2}-(1+\varepsilon)\left(u_{+}^{2}+u_{-}^{2}\right)\right. \\
& +(2 \lambda)^{-2}\left(u_{+}^{4}+u_{-}^{4}+6 u_{+}^{2} u_{-}^{2}\right)+\lambda^{2} \sin ^{2} \alpha\left(u_{+}^{2}+u_{-}^{2}\right) \\
& \left.+\sin \alpha\left(u_{-}^{3}+3 u_{+}^{3} u_{-}\right)\right] .
\end{aligned}
$$

Using $2 a b \geqq-c^{2} a^{2}-c^{-2} b^{2}$, we have

$$
\begin{aligned}
u_{+}^{2} u_{-} \sin \alpha & \geqq-\left\{(2 \lambda)^{-2} u_{+}^{4}+\lambda^{2} u_{-}^{2} \sin ^{2} \alpha\right\}, \\
2 u_{+}^{2} u_{-} \sin \alpha & \geqq-\left\{\frac{3}{2} \lambda^{-2} u_{+}^{2} u_{-}^{2}+\frac{2}{3} \lambda^{2} u_{+}^{2} \sin ^{2} \alpha\right\}, \\
u_{-}^{3} \sin \alpha & \geqq-\left\{\frac{1}{4} \lambda^{-2} u_{-}^{4}+\lambda^{2} u_{-}^{2} \sin ^{2} \alpha\right\} .
\end{aligned}
$$

Using the above inequalities in $D_{\varepsilon, \lambda}(u)$ yields

$$
\begin{aligned}
D_{\varepsilon, \lambda}(u)= & \int_{-\pi / 2}^{\pi / 2} d \alpha\left[\left(\frac{d u_{+}}{d \alpha}\right)^{2}+\left(\frac{d u_{-}}{d \alpha}\right)^{2}-(1+\varepsilon)\left(u_{+}^{2}+u_{-}^{2}\right)\right. \\
& \left.+\frac{1}{3} \lambda^{2} u_{+}^{2} \sin ^{2} \alpha-\lambda^{2} \sin ^{2} \alpha u_{-}^{2}\right] .
\end{aligned}
$$

The positive operator $A=-\left(d^{2} / d \alpha^{2}\right)+(1 / 3) \lambda^{2} \sin ^{2} \alpha$ is strictly greater than $-d^{2} / d \alpha^{2}$ since $\lambda^{2} \sin ^{2} \alpha$ is positive and the resolvent of $A$ is compact. 
The minimum eigenvalue of $-d^{2} / d^{2} \alpha$ with zero boundary conditions on $[-\pi / 2, \pi / 2]$ is 1 . Thus for some $\varepsilon_{+}>0$,

$$
\int_{-\pi / 2}^{\pi / 2} d \alpha\left[\left(\frac{d u_{+}}{d \alpha}\right)^{2}-(1+\varepsilon) u_{+}^{2}+\frac{1}{3} \lambda^{2} \sin ^{2} \alpha u_{+}^{2}\right] \geqq 0, \text { if } \varepsilon \leqq \varepsilon_{+} .
$$

The smallest eigenvalue of $-d^{2} / d \alpha^{2}$ with zero boundary conditions restricted to the odd subspace of $L^{2}((-\pi / 2, \pi / 2))$ is 4 , so for $\varepsilon \leqq 3 / 4$

$$
\begin{gathered}
\int_{-\pi / 2}^{\pi / 2} d \alpha\left[\left(\frac{d u_{-}}{d \alpha}\right)^{2}-(1+\varepsilon) u_{-}^{2}-i^{2} \sin ^{2} \alpha u_{-}^{2}\right] \\
\geqq \int_{-\pi / 2}^{\pi / 2} d \alpha\left[\left(\frac{d u_{-}}{d \alpha}\right)^{2}-\left(1+\lambda^{2}+\varepsilon\right) u_{-}^{2}\right] \geqq 0 .
\end{gathered}
$$

This completes the proof of Lemma 2.6 with $\varepsilon_{0}=\min \left\{\varepsilon_{+}, 3 / 4\right\}$.

Theorem 2.7. There are constants $a>0, b>0$ such that for $\phi \in B$

$$
A_{R}(\phi) \geqq a\left(\|\nabla \phi\|_{2}^{2}+\|\phi\|_{4, \mu}^{4}\right)-b .
$$

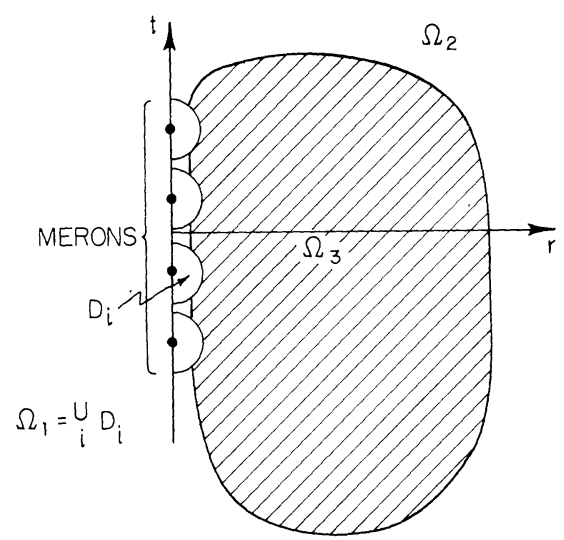

Fig. 1. The regions $\Omega_{1}, \Omega_{2}$ and $\Omega_{3}$

Proof. We divide the half plane $\mathbb{R}_{+}^{2}$ into three disjoint regions $\Omega_{1}, \Omega_{2}$, and $\Omega_{3}$ (see Fig. 1). Let $D_{i}=\left\{(r, t) \in \mathbb{R}_{+}^{2} \mid r^{2}+\left(t-t_{i}\right)^{2}<\delta^{2}\right\}$ and define

$$
\Omega_{1}=\bigcup_{i=1}^{2 n} D_{i}
$$

We assume $\delta \leqq 1 / 2 \min \left\{\mid t_{i}-t_{j} \| i \neq j\right\}$. Let $\varepsilon=\frac{\varepsilon_{0}}{40}$, with $\varepsilon_{0}$ as in Lemma 2.6 and define

$$
\Omega_{2}=\left\{x \in\left(\mathbb{R}_{+}^{2}-\Omega_{1}\right) \mid \Psi_{0}^{2}(x)>1-\varepsilon\right\} .
$$

Finally, $\Omega_{3}=\mathbb{R}_{+}^{2}-\left(\Omega_{1} \cup \Omega_{2}\right)$.

For $\Omega$ a measurable subset of $\mathbb{R}_{+}^{2}$ and $\phi \in C_{0}^{\infty}\left(\mathbb{R}_{+}^{2}\right)$ define

$$
E_{\Omega}(\phi)=\int_{\Omega} d^{2} x\left\{\left(\frac{1}{2}-8 \varepsilon\right)(\nabla \phi)^{2}+r^{-2}\left[\frac{1}{2}\left(3 \Psi_{0}^{2}-(1-4 \varepsilon)\right) \phi^{2}+\Psi_{0} \phi^{3}+\frac{1}{4} \phi^{4}\right]\right\} .
$$


Since $C_{0}^{\infty}\left(\mathbb{R}_{+}^{2}\right)$ is dense in $B$ it suffices to prove the theorem for $\phi \in C_{0}^{\infty}\left(\mathbb{R}_{+}^{2}\right)$. By Lemma 2.4,

$$
A_{R}(\phi) \geqq \sum_{i=1}^{3} E_{\Omega_{1}}(\phi)+\int_{\mathbb{R}_{+}^{2}} d^{2} x f \phi .
$$

We bound each $E_{\Omega_{1}}(\phi)$ from below. Consider first a half disc $D_{i} \subset \Omega_{1}$ and introduce polar coordinates $(\varrho, \alpha)$ in $D_{i}$ :

$$
\begin{array}{ll}
r=\varrho \cos \alpha, & t=t_{i}+\varrho \sin \alpha \\
0<\varrho<\delta, & -\pi / 2<\alpha<\pi / 2 .
\end{array}
$$

Note that in the disc $D_{i}$,

$$
\Psi_{0}= \pm \sin \alpha+h(\varrho, \alpha),
$$

where $\left\langle h \|_{\infty, D_{\imath}} \rightarrow 0\right.$ as $\delta \rightarrow 0$.

Hence,

$$
\begin{aligned}
E_{D_{i}}(\phi) \geqq & \int_{D_{2}} d^{2} x\left\{\left(\frac{1}{2}-8 \varepsilon\right)(\nabla \phi)^{2}+r^{-2}\left[\frac{1}{2}\left(3 \sin ^{2} \alpha-(1-4 \varepsilon)\right) \phi^{2}\right.\right. \\
& \left.\left.-\left|(\sin \alpha) \phi^{3}\right|+\frac{1}{4} \phi^{4}-3|h \sin \alpha| \phi^{2}-\left|h \phi^{3}\right|\right]\right\}
\end{aligned}
$$

We choose $\delta$ so small that $\|h\|_{\infty, D_{2}} \leqq \frac{\varepsilon}{3}$ for any $i \in\{1, \ldots, 2 n\}$. Lemma 2.5 with $a=1 / 2$ implies

$$
\int_{D_{l}} d^{2} x\left[4 \varepsilon(\nabla \phi)^{2}-3 r^{-2}|h \sin \alpha| \phi^{2}\right] \geqq 0,
$$

while from the Schwarz inequality and Lemma 2.5 we have

$$
\int_{D_{i}} d^{2} x r^{-2}\left|h \phi^{3}\right| \leqq 2\|h\|_{\infty, D_{t}}\|\nabla \phi\|_{2, D_{\imath}}\|\phi\|_{4, \mu, D_{i}}^{2} .
$$

Consequently,

$$
\begin{aligned}
E_{D_{l}}(\phi) \geqq & \int_{D_{\imath}} d^{2} x\left\{\left(\frac{1}{2}-12 \varepsilon\right)(\nabla \phi)^{2}+r^{-2}\left[\frac{1}{2}\left(3 \sin ^{2} \alpha-(1-4 \varepsilon)\right) \phi^{2}\right.\right. \\
& \left.\left.-\left|(\sin \alpha) \phi^{3}\right|+\frac{1}{4} \phi^{4}\right]\right\}-\frac{2}{3} \varepsilon\|\nabla \phi\|_{2, D_{\imath}}\|\phi\|_{4, \mu, D_{\imath}}^{2} .
\end{aligned}
$$

Now, with $v=(1+2 \varepsilon)^{1 / 2}$ and

$$
\sigma=\frac{1}{4}\left(1-v^{-2}\right)=\frac{\varepsilon}{2+4 \varepsilon}
$$

we have

$$
\begin{aligned}
& \frac{1}{2}\left(3 \sin ^{2} \alpha-(1-4 \varepsilon)\right) \phi^{2}-\left|\sin \alpha \phi^{3}\right|+\frac{1}{4} \phi^{4} \\
& =\left(v|(\sin \alpha) \phi|-\frac{1}{2} v^{-1} \phi^{2}\right)^{2}+\sigma \phi^{4}-\frac{1}{2}(1-4 \varepsilon)\left(\cos ^{2} \alpha\right) \phi^{2} .
\end{aligned}
$$

Inserting the above identity into (2.2) and using $r \leqq \varrho$ yields

$$
\begin{aligned}
E_{D_{l}}(\phi) \geqq & \int_{D_{\imath}} \varrho d \varrho d \alpha\left\{\left(\frac{1}{2}-12 \varepsilon\right)(\nabla \phi)^{2}-\left(\frac{1}{2}-2 \varepsilon\right) \varrho^{-2} \phi^{2}\right. \\
& \left.+\varrho^{-2} \phi^{2}\left(v|\sin \alpha|-(2 v)^{-1}|\phi|\right)^{2}\right\}+\sigma\|\phi\|_{4, \mu, D_{l}}^{4} \\
& -\frac{2}{3} \varepsilon\|\nabla \phi\|_{2, D_{i}}\|\phi\|_{4, \mu, D_{i}}^{2} .
\end{aligned}
$$


By the definition of $\varepsilon$ and the fact that $\varepsilon_{0} \leqq 3 / 4$ one obtains

$$
v \in(1,3 / 2)
$$

and

$$
\left(\frac{1}{2}-2 \varepsilon\right)\left(\frac{1}{2}-24 \varepsilon\right)^{-1} \leqq 1+\varepsilon_{0} .
$$

Thus, we can apply Lemma 2.6 to conclude

$$
\int_{D_{2}} \varrho d \varrho d \alpha\left\{\left(\frac{1}{2}-24 \varepsilon\right)(\nabla \phi)^{2}-\left(\frac{1}{2}-2 \varepsilon\right) \varrho^{-2} \phi^{2}+Q^{-2} \phi^{2}\left(v|\sin \alpha|-(2 v)^{-1}|\phi|\right)^{2}\right\} \geqq 0 .
$$

Hence,

$$
\begin{aligned}
E_{D_{\imath}}(\phi) & \geqq 12 \varepsilon\|\nabla \dot{\phi}\|_{2, D_{i}}^{2}+\sigma\|\phi\|_{4, \mu, D_{\imath}}^{4}-\frac{2}{3} \varepsilon\|\nabla \phi\|_{2, D_{\imath}}\|\phi\|_{4, \mu, D_{\imath}}^{2} \\
& \geqq \frac{\varepsilon}{4}\left(\|\nabla \phi\|_{2, D_{\imath}}^{2}+\|\phi\|_{4, \mu, D_{\imath}}^{4}\right) .
\end{aligned}
$$

This argument can be repeated in each disc $D_{i}$ so

$$
E_{\Omega_{1}}(\phi) \geqq \frac{\varepsilon}{4}\left(\|\nabla \phi\|_{2, \Omega_{1}}^{2}+\|\phi\|_{4, \mu, \Omega_{1}}^{4}\right) .
$$

Next we consider region $\Omega_{2}$, where $\Psi_{0}^{2} \geqq 1-\varepsilon$. Since $\left|\Psi_{0}\right| \leqq 1$,

$$
\begin{aligned}
E_{\Omega_{2}}(\phi) & \geqq \int_{\Omega_{2}} d^{2} x\left\{\left(\frac{1}{2}-8 \varepsilon\right)(\nabla \phi)^{2}+r^{-2}\left[\left(1+\frac{\varepsilon}{2}\right) \phi^{2}-|\phi|^{3}+\frac{1}{4} \phi^{4}\right]\right\} \\
& \geqq\left(\frac{1}{2}-8 \varepsilon\right)\|\nabla \phi\|_{2, \Omega_{2}}^{2}+\frac{1}{4}\left[1-\left(1+\frac{\varepsilon}{2}\right)^{-1}\right]\|\phi\|_{4, \mu, \Omega_{2}}^{4} \\
& \geqq \frac{\varepsilon}{9}\left(\|\nabla \phi\|_{2, \Omega_{2}}^{2}+\|\phi\|_{4, \mu, \Omega_{2}}^{4}\right) .
\end{aligned}
$$

Finally,

$$
\begin{aligned}
E_{\Omega_{3}}(\phi) \geqq & \left(\frac{1}{2}-8 \varepsilon\right)\|\nabla \phi\|_{2, \Omega_{3}}^{2}-\frac{1}{2}\|\phi\|_{2, \mu, \Omega_{3}}^{2} \\
& -\|\phi\|_{2, \mu, \Omega_{3}}\|\phi\|_{4, \mu, \Omega_{3}}^{2}+\frac{1}{4}\|\phi\|_{4, \mu, \Omega_{3}}^{4} \\
\geqq & \left(\frac{1}{2}-8 \varepsilon\right)\|\nabla \phi\|_{2, \Omega_{3}}^{2}+\frac{1}{8}\|\phi\|_{4, \mu, \Omega_{3}}^{4}-\frac{5}{2}\|\phi\|_{2, \mu, \Omega_{3}}^{2} .
\end{aligned}
$$

Applying the Schwarz and Minkowski inequalities we have

$$
\|\phi\|_{2, \mu, \Omega_{3}}^{2} \leqq \mu\left(\Omega_{3}\right)^{1 / 2}\|\phi\|_{4, \mu, \Omega_{3}}^{2} \leqq \frac{1}{40}\|\phi\|_{4, \mu, \Omega_{3}}^{4}+10 \mu\left(\Omega_{3}\right)
$$

so

$$
E_{\Omega_{3}}(\phi) \geqq \frac{1}{16}\left(\|\nabla \phi\|_{2, \Omega_{3}}^{2}+\|\phi\|_{4, \mu, \Omega_{3}}^{4}\right)-25 \mu\left(\Omega_{3}\right) .
$$

Now, $\|r f\|_{2}<\infty$ (see Appendix), so

$$
\left|\int_{\mathbb{R}_{+}^{2}} d^{2} x f \phi\right| \leqq\|r f\|_{2}\|\phi\|_{2, \mu} \leqq 2\|r f\|_{2}\|\nabla \phi\|_{2},
$$

by Lemma 2.4 .

Combining (2.1) and (2.3)-(2.6), there are constants $a>0, b>0$ such that $A_{R}(\phi)$ $\geqq a\left(\|\phi\|_{2}^{2}+\|\phi\|_{4, \mu}^{4}\right)-b$. This completes the proof. 
Corollary 2.8. The estimate E1 of Sect. I is valid.

Proof. This is an immediate consequence of Theorem 2.7.

Proposition 2.9. Let $\Omega \subset \mathbb{R}_{+}^{2}$ be open and $\bar{\Omega}$ compact in $\mathbb{R}_{+}^{2}$. Then there is a function $\phi_{\Omega} \in B_{\Omega}$ that solves (1.4) weakly in the region $\Omega$.

Proof. By Proposition 2.1, Theorem 2.3, and Corollary 2.8 there is a function $\phi_{\Omega} \in B_{\Omega}$ that minimizes $A_{R}$ on $B_{\Omega}$. Let $w \in C_{0}^{\infty}(\Omega)$. Since

$$
A_{R}\left(\phi_{\Omega}+w\right) \geqq A_{R}\left(\phi_{\Omega}\right)
$$

the term in $A_{R}\left(\phi_{\Omega}+w\right)$ that is linear in $w$ vanishes. Hence,

$$
\int_{\Omega} d^{2} x\left\{-\phi_{\Omega} \Delta w+w f+w r^{-2}\left[\left(3 \Psi_{0}^{-2}-1\right) \phi_{\Omega}+3 \Psi_{0} \phi_{\Omega}^{2}+\phi_{\Omega}^{3}\right]\right\}=0 .
$$

Let us denote $\phi_{M_{n}}$ by $\phi_{n}$.

Proposition 2.10. The sequence $\left\{\phi_{n}\right\}_{n=1}^{\infty}$ has a weakly convergent subsequence in $B$.

Proof. The Banach space $B$ is obviously separable. It is reflexive, being isometrically isomorphic to a closed subspace of $L^{2}\left(\mathbb{R}_{+}^{2}\right)$ under the mapping $\phi \rightarrow|\nabla \phi|$. It follows that bounded sets in $B$ are weakly precompact so it suffices to show that $\left\{\phi_{n}\right\}_{n=1}^{\infty}$ is bounded in $B$.

By Theorem 2.7,

$$
\left\|\phi_{n}\right\|_{B}^{2} \leqq a^{-1}\left(A_{R}\left(\phi_{n}\right)+b\right) \text { for any } n \text {. }
$$

On the other hand

$$
A_{R}\left(\phi_{n}\right)=\inf _{\phi \in B_{n}} A_{R}(\phi) \leqq \inf _{\phi \in B_{1}} A_{R}(\phi)=A_{R}\left(\phi_{1}\right),
$$

since $B_{1} \subseteq B_{n}$ for any $n \geqq 1$. Hence, $\left\|\phi_{n}\right\|_{B}^{2} \leqq a^{-1}\left(A_{R}\left(\phi_{1}\right)+b\right)$

for any $n \geqq 1$.

We can assume without loss of generality that $\left\{\phi_{n}\right\}_{n=1}^{\infty}$ is weakly convergent. Let $\Phi$ denote its weak limit.

Theorem 2.11. The function $\Phi \in B$ is a weak solution to (1.4) in $\mathbb{R}_{+}^{2}$.

Proof. Let $w \in C_{0}^{\infty}\left(\mathbb{R}_{+}^{2}\right)$. Choose $n_{0}$ so large that suppwc $M_{n_{0}}$. Then, by Proposition 2.9,

$$
\int_{M_{n}} d^{2} x\left\{-\phi_{n} \Delta w+w f+w r^{-2}\left[\left(3 \Psi_{0}^{2}-1\right) \phi_{n}+3 \Psi_{0} \phi_{n}^{2}+\phi_{n}^{3}\right]\right\}=0
$$

for all $n \geqq n_{0}$. Now let $n \rightarrow \infty$. Since $\left\{\phi_{n}\right\}$ converges weakly to $\Phi$ in $B,\left\{\phi_{n} w\right\}$ converges weakly to $\Phi_{w}$ in $B_{n_{0}}$. The imbedding

$$
B_{n_{0}} \rightarrow L^{p}\left(M_{n_{0}}\right)
$$

is compact for any $p \in[1, \infty)$ (see e.g. [9]), so $\left\{w \phi_{n}^{p}\right\}$ converges strongly to $w \Phi^{p}$ in $L^{1}\left(M_{n_{0}}\right)$. Since $\Psi_{0}$ and $r^{-2}$ are bounded on $M_{n_{0}}$, it follows that

$$
\int_{\mathbb{R}_{+}^{2}} d^{2} x\left\{-\Phi \Delta w+w f+w r^{-2}\left[\left(3 \Psi_{0}^{2}-1\right) \Phi+3 \Psi_{0} \Phi^{2}+\Phi^{3}\right]\right\}=0 .
$$




\section{Regularity of Solutions}

We begin by proving interior regularity of the weak solution $\Phi$ obtained in Theorem 2.11 which follows by standard estimates. Regularity at the boundary follows from an a priori estimate on $\nabla \Phi$.

Theorem 3.1. The function $\Phi$ is real analytic in $\mathbb{R}_{+}^{2}$.

Proof. Let $\Omega$ be a compact subset of $\mathbb{R}_{+}^{2}$ with a regular boundary. Since $\Phi$ is a weak solution of (1.4), $\Delta \Phi \in L^{2}(\Omega)$. Thus $\Phi$ is in the Sobolev space $W^{2,2}(\Omega)$. By Sobolev's Lemma ([9, p. 97]), $\Phi \in C^{0}(\Omega)$. Repeating this argument for derivatives of $\Phi$ we obtain $\Phi \in C^{3}(\Omega)$. By a standard theorem it follows that $\Phi$ is real analytic ([10, pp. $170-180]$ ) in $\Omega$.

To show that $\Phi$ tends continuously to zero at the boundary of $\mathbb{R}_{+}^{2}$ we prove a simple property of the Banach space $B$.

Theorem 3.2. Let $\phi \in B$ with $\|r \nabla \phi\|_{\infty}<c$. Then

$$
\lim _{r \rightarrow 0} \phi(x)=\lim _{x \rightarrow \infty} \phi(x)=0 .
$$

Proof. Suppose on the contrary that there is a sequence $x_{n} \in \mathbb{R}_{+}^{2}$ with $\left|\phi\left(x_{n}\right)\right|>\varepsilon$ and either $r_{n} \rightarrow 0$ or $\left|x_{n}\right| \rightarrow \infty$. Choosing a subsequence, if necessary, we may assume that discs of radius $r_{n} / 2$ about $x_{n}$ are non-overlapping. Let $D_{n}$ be the disc of radius $\delta r_{n}$ about $x_{n}$, where $\delta=\min \{1 / 2, \varepsilon / 4 c\}$. In $D_{n}, r \geqq r_{n} / 2$ so $\|\nabla \phi\|_{\infty, D_{n}} \geqq 2 c r_{n}^{-1}$. Thus for $x \in D_{n}$ and $L$ a straight line from $x_{n}$ to $x$,

$$
\begin{aligned}
|\phi(x)| & =\left|\phi\left(x_{n}\right)+\int_{L} \nabla \phi\left(x^{\prime}\right) \cdot d l^{\prime}\right| \\
& \geqq \varepsilon-2 c r_{n}^{-1} \cdot(\varepsilon / 4 c) r_{n}=\varepsilon / 2 .
\end{aligned}
$$

This contradicts the assumption that $\phi \in B$ since it implies that

$$
\begin{aligned}
\|\phi\|_{B}^{2} & \geqq \frac{1}{4} \int_{\mathbb{R}_{+}^{2}} d^{2} x r^{-2} \phi^{2} \geqq \frac{1}{4} \sum_{n} \int_{D_{n}} d^{2} x r^{-2} \phi^{2} \\
& \geqq \frac{1}{4} \sum_{n} \pi\left(\delta r_{n}\right)^{2}\left(3 r_{n} / 2\right)^{-2}(\varepsilon / 2)^{2}=\infty .
\end{aligned}
$$

Lemma 3.3. Let $\psi \in C^{2}\left(\mathbb{R}_{+}^{2}\right)$ be a solution of $r^{2} \Delta \psi=\psi^{3}-\psi$ in $\mathbb{R}_{+}^{2}$. Then

$$
\|r \nabla \psi\|_{\infty} \leqq \frac{4}{3}\left\|\psi^{3}-\psi\right\|_{\infty}+4\|\psi\|_{\infty} .
$$

Proof. To estimate $\nabla \psi(\mathbf{x}), \mathbf{x}=(r, t) \in \mathbb{R}_{+}^{2}$, we use the Dirichlet Green's function $g$ for Laplace's equation in the closed disc $D$ of radius $r / 2$ and center $\mathbf{x}$ :

$$
g\left(\mathbf{x}^{\prime}, \mathbf{x}^{\prime \prime}\right)=(2 \pi)^{-1}\left\{\ln \left|\mathbf{x}^{\prime}-\mathbf{x}^{\prime \prime} *\right|-\ln \left|\mathbf{x}^{\prime}-\mathbf{x}^{\prime \prime}\right|-\ln \frac{r}{2}+\ln \left|\mathbf{x}^{\prime \prime}-\mathbf{x}\right|\right\},
$$

where $\mathbf{x}^{\prime \prime}=\mathbf{x}+\frac{\mathbf{x}^{\prime \prime}-\mathbf{x}}{\left|\mathbf{x}^{\prime \prime}-\mathbf{x}\right|^{2}}\left(\frac{r}{2}\right)^{2}$. The function $\psi$ satisfies the identity

$$
\begin{aligned}
\psi\left(\mathbf{x}^{\prime}\right)= & \int_{D} d^{2} x^{\prime \prime} g\left(\mathbf{x}^{\prime}, \mathbf{x}^{\prime \prime}\right) r^{\prime \prime}-2\left(\psi^{3}\left(\mathbf{x}^{\prime \prime}\right)-\psi\left(\mathbf{x}^{\prime \prime}\right)\right) \\
& -\int_{C D} d s^{\prime \prime} \frac{\partial g\left(\mathbf{x}^{\prime}, \mathbf{x}^{\prime \prime}\right)}{\partial n^{\prime \prime}} \psi\left(\mathbf{x}^{\prime \prime}\right),
\end{aligned}
$$


where $\partial / \partial n^{\prime \prime}$ denotes the outward normal derivative. Differentiating (3.2) with respect to $\mathbf{x}^{\prime}$ and setting $\mathbf{x}^{\prime}=\mathbf{x}$ we obtain

$$
\nabla g\left(\mathbf{x}, \mathbf{x}^{\prime \prime}\right)=\frac{1}{2 \pi}\left(\left|\mathbf{x}-\mathbf{x}^{\prime \prime}\right|^{-2}-4 r^{-2}\right)\left(\mathbf{x}^{\prime \prime}-\mathbf{x}\right)
$$

and differentiating with respect to $\mathbf{x}^{\prime \prime}$ gives

$$
\left.\nabla \frac{\partial g}{\partial n^{\prime \prime}}\left(\mathbf{x}, \mathbf{x}^{\prime \prime}\right)\right|_{\mathbf{x}^{\prime \prime} \in \hat{C} D}=8 \pi^{-1} r^{-3}\left(\mathbf{x}^{\prime \prime}-\mathbf{x}\right) \text {. }
$$

Thus, differentiating (3.3) at $\mathbf{x}^{\prime}=\mathbf{x}$ and taking sup norms yields

$$
|\nabla \psi(x)| \leqq \frac{4}{3} r^{-1}\left\|\psi^{3}-\psi\right\|_{\infty, D}+4 r^{-1}\|\psi\|_{\infty, i D},
$$

where we have used $r^{\prime \prime} \geqq r / 2$ for $\mathbf{x}^{\prime \prime} \in D$.

Let us denote $\phi_{n}+\Psi_{0}$ by $\psi_{n}$ and let $\Psi \equiv \Psi_{0}+\Phi$.

Lemma 3.4. The function $\psi_{n}$ satisfies $\left\|\psi_{n}\right\|_{\infty, M_{n}} \leqq 1$.

Proof. Suppose there is $x_{0} \in M_{n}$ such that $\left|\psi_{n}\left(x_{0}\right)\right|>1$. By the argument of Theorem $3.1 \psi_{n}$ is real analytic in $M_{n}$ so there is a neighborhood of $x_{0}$ where $\left|\psi_{n}\right|>1$.

Let $F \in C^{1}(\mathbb{R})$ satisfy

i) $F(\xi)=\xi$ if $|\xi| \leqq 1$,

ii) $|F(\xi)|<|\xi|$ if $|\xi|>1$,

iii) $\left.\mid F^{\prime}(\xi)\right) \leqq 1$ for all $\xi \in \mathbb{R}$.

It follows from i), ii), and iii) that $A_{M_{n}}\left(F \circ \psi_{n}\right)<A_{M_{n}}\left(\psi_{n}\right)$. We have $A_{R}\left(\phi_{n}\right)$ $=A_{M_{n}}\left(\psi_{n}\right)-A_{M_{n}}\left(\Psi_{0}\right)$ so defining $\tilde{\phi}_{n}=F \circ \psi_{n}-\Psi_{0}$ one obtains

$$
A_{R}\left(\tilde{\phi}_{n}\right)<A_{R}\left(\phi_{n}\right) \text {. }
$$

We claim that $\tilde{\phi}_{n} \in B_{n}$, which contradicts the fact that $\phi_{n}$ minimizes $A_{R}$ on $B_{n}$, and the lemma follows. To verify the claim we use a trace theorem for Sobolev spaces (see e.g. $[9$, p. 216]). The mapping

$$
\gamma: W^{1.2}\left(M_{n}\right) \rightarrow W^{1 / 2,2}\left(\hat{c} M_{n}\right)
$$

defined by $\gamma(u)=u \uparrow \hat{\partial} M_{n}$ is continuous. The kernel of $\gamma$ is precisely $W_{0}^{1,2}\left(M_{n}\right)=B_{n}$. Now, $\phi_{n} \in B_{n}$ so $\phi_{n} \uparrow \partial M_{n}=0$ a.e. Hence, $\tilde{\phi}_{n} \uparrow \partial M_{n}=0$ a.e. and $\tilde{\phi}_{n} \in W^{1,2}\left(M_{n}\right)$ by (3.4) so $\gamma\left(\tilde{\phi}_{n}\right)=0$ in $W^{1 / 2,2}\left(\partial M_{n}\right)$ which implies $\tilde{\phi}_{n} \in B_{n}$.

Theorem 3.5. The function $\Psi$ satisfies $\|\Psi\|_{\infty} \leqq 1$.

Proof. Suppose there is $x_{0} \in \mathbb{R}_{+}^{2}$ such that $\left|\Psi\left(x_{0}\right)\right|>1$. Then there is a neighborhood, $V$, of $x_{0}$ and $\varepsilon>0$ such that $|\Psi(x)|>1+\varepsilon$, for all $x \in V$. Let $g \in C_{0}^{\infty}(V), g \geqq 0$, $g \neq 0$. Since $\left\{\phi_{n}\right\}$ converges weakly to $\Phi$, we have

$$
\int_{V} g \phi_{n} d^{2} x \rightarrow \int_{V} g \Phi d^{2} x \quad \text { as } n \rightarrow \infty,
$$

and consequently

$$
\int_{V} g\left(\psi_{n}-\Psi\right) d^{2} x \rightarrow 0 \quad \text { as } \quad n \rightarrow \infty
$$


This is impossible, since $\left\|\psi_{n}\right\|_{\infty} \leqq 1$ which implies $\left\{\psi_{n}-\Psi \mid>\varepsilon\right.$ on $V$.

Theorem 3.6. The function $\Phi$ satisfies $\|r \nabla \Phi\|_{\infty}<2 n+6$, where $2 n$ is the number of merons.

Proof. We apply Lemma 3.3 and Theorem 3.5 to $\Psi$ obtaining

$$
\|r \nabla \Psi\|_{\infty} \leqq \frac{4}{3}\left\|\Psi^{3}-\Psi\right\|_{\infty}+4\|\Psi\|_{\infty}<6 .
$$

Theorem 3.6 follows immediately, since $\left\|r \nabla \Psi_{0}\right\|_{\infty} \leqq 2 n$.

Corollary 3.7. The function $\Phi$ satisfies

$$
\lim _{r \rightarrow 0} \Phi(x)=\lim _{x \rightarrow \infty} \Phi(x)=0 .
$$

Theorem 1.1 of the Introduction has now been proven. It is a restatement of Theorems 2.11, 3.1, 3.2, 3.6, and Corollary 3.7 for $\Psi \equiv \Psi_{0}+\Phi$.

Acknowledgement. We would like to acknowledge helpful conversations with Arthur Jaffe and (in the case of J.H., O.McB.) with Lars Wahlbin. We are also indebted to Bradley Plohr for pointing out an error in the original version of this paper, and to David Groisser for careful reading of the manuscript.

\section{Appendix}

Lemma A1. Let $f$ be defined by (1.6) and (1.9).

Then,

i) $f$ is $O\left(\left(r^{2}+t^{2}\right)^{-2}\right)$ at infinity,

ii) $f$ is bounded,

iii) $r f \in L^{2}\left(\mathbb{R}_{+}^{2}\right)$.

Proof. We have $\Psi_{0}=\cos \theta$, where $\theta=\sum_{i=1}^{2 n}(-1)^{i} \theta_{i}, \theta_{i}=\arctan \left(\frac{t-t_{i}}{r}\right)$.

Let $\hat{\mathbf{r}}, \hat{\mathbf{t}}$ be unit vectors in the $r$ and $t$ directions, respectively. Then

$$
\nabla \theta_{i}=\left[r^{2}+\left(t-t_{i}\right)^{2}\right]^{-1}\left(r \hat{\mathbf{t}}-\left(t-t_{i}\right) \hat{\mathbf{r}}\right)
$$

and

$$
\Delta \theta_{i}=0 .
$$

Hence,

$$
\Delta \Psi_{0}=-\Psi_{0}(\nabla \theta)^{2}
$$

so

$$
f=\Psi_{0}\left((\nabla \theta)^{2}-\frac{\sin ^{2} \theta}{r^{2}}\right) .
$$

By the mean value theorem,

$$
\left|\theta_{2 i}(r, t)-\theta_{2 i-1}(r, t)\right| \leqq|\Delta t| \sup _{t^{\prime} \in[t, t+\Delta t]}\left|\frac{\partial}{\partial t^{\prime}} \theta_{2 i}\left(r, t^{\prime}\right)\right|,
$$

where $\Delta t=t_{2 i}-t_{2 i-1}$. Denote $\left(r^{2}+t^{2}\right)^{1 / 2}$ by $R$. Then for $R$ large

$$
\left|\theta_{2 i}(r, t)-\theta_{2 i-1}(r, t)\right| \leqq O(1) r R^{-2} \text {. }
$$


Similarly, for $R$ large,

$$
\left|\nabla \theta_{2 i}(r, t)-\nabla \theta_{2 i-1}(r, t)\right| \leqq O(1) R^{-2} .
$$

It follows that

$$
\begin{aligned}
\left|r^{-2} \sin ^{2} \theta-(\nabla \theta)^{2}\right|= & \left|r^{-2} \theta^{2}-(\nabla \theta)^{2}\right|+O\left(R^{-6}\right) \\
= & \mid \sum_{i=1}^{n} \sum_{j=1}^{n} r^{-2}\left(\theta_{2 i}-\theta_{2 i-1}\right)\left(\theta_{2 j}-\theta_{2 j-1}\right) \\
& -\left(\nabla \theta_{2 i}-\nabla \theta_{2 i-1}\right) \cdot\left(\nabla \theta_{2 j}-\nabla \theta_{2 j-1}\right) \mid+O\left(R^{-6}\right)=O\left(R^{-4}\right) .
\end{aligned}
$$

Since $\left|\Psi_{0}\right| \leqq 1$, this proves $\left.i\right)$.

Let $\varepsilon>0$,

$$
B_{i}=\left\{(r, t) \in \mathbb{R}_{+}^{2} \mid r^{2}+\left(t-t_{i}\right)^{2}<\varepsilon^{2}\right\}
$$

and

$$
U=\bigcup_{i=1}^{2 n} B_{i}
$$

We see from (A1) that $(\nabla \theta)^{2}$ is bounded on the complement of $U$. Furthermore, $\theta(r, t)=O(r)$ for $r$ small and $(r, t) \in \mathbb{R}_{+}^{2}-U$ so $f$ is bounded on the complement of $U$.

Now focus attention on one of the half discs $B_{i} \subset U$ and assume $\varepsilon<\min _{i \neq j}\left|t_{i}-t_{j}\right|$. Then $\theta=\theta_{i}+\xi$ where $\xi$ has a continuous extension to the closure of $B_{i}$ in $\mathbb{R}^{2}$. We observe that $(\nabla \xi)^{2}$ is bounded on $B_{i}$. Now introduce polar coordinates in $B_{i}$ so that $\theta_{i}$ is the polar angle. Let $\varrho$ denote the radial coordinate. Then

$$
\begin{aligned}
r^{-2} \sin ^{2} \theta= & \left(\varrho^{2} \cos ^{2} \theta_{i}\right)^{-2} \times\left(\sin ^{2} \theta_{i} \cos ^{2} \xi+\cos ^{2} \theta_{i} \sin ^{2} \xi\right. \\
& \left.+2 \sin \theta_{i} \cos \theta_{i} \sin \xi \cos \xi\right)
\end{aligned}
$$

and

$$
\left(\nabla \theta_{i}\right)^{2}+2 \nabla \theta_{i} \cdot \nabla \xi=Q^{-2}+2 \sum_{j \neq i} \frac{r^{2}+\left(t-t_{i}\right)\left(t-t_{j}\right)}{\varrho^{2}\left(r^{2}+\left(t-t_{j}\right)^{2}\right)} .
$$

To prove that $f$ is bounded on $B_{i}$ we therefore need to show that

$$
\begin{gathered}
\tan ^{2} \theta_{i} \cos ^{2} \xi+\sin ^{2} \xi+2 \tan \theta_{i} \sin \xi \cos \xi-1 \\
\quad-2 \sum_{j \neq i} \frac{r^{2}+\left(t-t_{i}\right)\left(t-t_{j}\right)}{r^{2}+\left(t-t_{j}\right)^{2}}=O\left(\varrho^{2}\right) .
\end{gathered}
$$

By Taylor's formula

$$
\sin ^{2} \xi=1+O\left(r^{2}\right), \quad \cos ^{2} \xi=O\left(r^{2}\right)
$$

so we only need to show

$$
\tan \theta_{i} \sin \xi \cos \xi-\sum_{j \neq i} \frac{r^{2}+\left(t-t_{i}\right)\left(t-t_{j}\right)}{r^{2}+\left(t-t_{j}\right)^{2}}=O\left(\varrho^{2}\right) .
$$


We have

$$
\begin{aligned}
\tan \theta_{i} & =\frac{t-t_{i}}{r} \text { and } \sin \xi \cos \xi \\
& =\sin \left( \pm \frac{\pi}{2}-\sum_{j \neq i} \arctan \frac{r}{t-t_{j}}\right) \cos \left( \pm \frac{\pi}{2}-\sum_{j \neq i} \arctan \frac{r}{t-t_{j}}\right) \\
& =\sum_{j \neq i} \frac{r}{t-t_{j}}+O\left(\varrho^{2}\right),
\end{aligned}
$$

so the left hand side of (A2) becomes

$$
\sum_{j \neq i}\left[\frac{t-t_{i}}{t-t_{j}}-\frac{r^{2}+\left(t-t_{i}\right)\left(t-t_{j}\right)}{r^{2}+\left(t-t_{j}\right)^{2}}\right]+O\left(Q^{2}\right)=O\left(\varrho^{2}\right) .
$$

This proves ii) as the above argument applies in each of the discs $B_{i}$.

Finally, i) and ii) imply iii) since

$$
\begin{aligned}
\int_{\mathbb{R}_{+}^{2}} r^{2} f^{2} d^{2} x & =\int_{R \leqq R_{0}} r^{2} f^{2} d^{2} x+\int_{R \geqq R_{0}} r^{2} f^{2} d^{2} x \\
& \leqq\|f\|_{\infty}^{2} \int_{R \leqq R_{0}} r^{2} d^{2} x+O(1) \int_{R \geqq R_{0}} \frac{r^{2}}{\left(r^{2}+t^{2}\right)^{4}} d^{2} x
\end{aligned}
$$

and both the integrals above are finite.

Lemma A2. Let $\Omega \subset \mathbb{R}_{+}^{2}$ be open and $\bar{\Omega}$ compact in $\mathbb{R}_{+}^{2}$. Then $A_{R}$ is well defined and strongly continuous on $B_{\Omega}$.

Proof. Let $\phi \in B_{\Omega}$. Then there is a sequence $\left\{\phi_{n}\right\}_{n=1}^{\infty} \subset C_{0}^{\infty}(\Omega)$ converging to $\phi$ in the $B_{\Omega}$-norm. We shall verify that $A_{R}\left(\phi_{n}\right)$ is Cauchy in $\mathbb{R}$. We have

$$
\begin{aligned}
\left|A_{R}\left(\phi_{n}\right)-A_{R}\left(\phi_{m}\right)\right|= & \mid \int_{\mathbb{R}_{+}^{2}} d^{2} x\left\{\frac{1}{2}\left(\nabla \phi_{n}\right)^{2}-\frac{1}{2}\left(\nabla \phi_{m}\right)^{2}+f\left(\phi_{n}-\phi_{m}\right)\right. \\
& \left.+r^{-2}\left[\frac{3 \Psi_{0}^{2}-1}{2}\left(\phi_{n}^{2}-\phi_{m}^{2}\right)+\Psi_{0}\left(\phi_{n}^{3}-\phi_{m}^{3}\right)+\frac{1}{4}\left(\phi_{n}^{4}-\phi_{m}^{4}\right)\right]\right\} \mid \\
\leqq & \frac{1}{2}\left\|\nabla \phi_{n}+\nabla \phi_{m}\right\|_{2, \Omega}\left\|\nabla \phi_{n}-\nabla \phi_{m}\right\|_{2, \Omega}+\|f\|_{2, \Omega}\left\|\phi_{n}-\phi_{m}\right\|_{2} \\
& +\frac{1}{2}\left\|\left(3 \Psi_{0}^{2}-1\right) r^{-2}\right\|_{\infty, \Omega}\left\|\phi_{n}+\phi_{m}\right\|_{2, \Omega}\left\|\phi_{n}-\phi_{m}\right\|_{2, \Omega} \\
& +\left\|r^{-2} \Psi_{0}\right\|_{\infty, \Omega}\left\|\left(\phi_{n}^{2}+\phi_{m} \phi_{n}+\phi_{m}^{2}\right)\right\|_{2, \Omega}\left\|\phi_{n}-\phi_{m}\right\|_{2, \Omega} \\
& +\frac{1}{4}\left\|\left(\phi_{n}^{3}+\phi_{n}^{2} \phi_{m}+\phi_{n} \phi_{m}^{2}+\phi_{m}^{3}\right)\right\|_{2, \Omega}\left\|\phi_{n}-\phi_{m}\right\|_{2, \Omega}\left\|r^{-2}\right\|_{\infty, \Omega} .
\end{aligned}
$$

There is a constant $C$, such that

$$
\|\nabla \phi\|_{2, \Omega} \geqq C\|\phi\|_{2, \Omega}
$$

for all $\phi \in B_{\Omega}$. Hence,

$$
\left|A_{R}\left(\phi_{n}\right)-A_{R}\left(\phi_{m}\right)\right| \leqq \text { const }\left\|\phi_{n}-\phi_{m}\right\|_{B}
$$

since $\left\|\nabla \phi_{n}+\nabla \phi_{m}\right\|_{2 . \Omega},\left\|\left(\phi_{n}+\phi_{m}\right)\right\|_{2, \Omega}$,

$$
\left\|\left(\phi_{n}^{2}+\phi_{n} \phi_{m}+\phi_{m}^{2}\right)\right\|_{2, \Omega} \text { and }\left\|\left(\phi_{n}^{3}+\phi_{n}^{2} \phi_{m}+\phi_{n} \phi_{m}^{2}+\phi_{m}^{3}\right)\right\|_{2, \Omega}
$$

are all uniformly bounded. 


\section{References}

1. Belavin, A., Polyakov, A., Schwartz, A., Tyupkin, Y.: Phys. Lett. 59B, 85 (1975)

2. Witten, E. : Phys. Rev. Lett. 38, 121 (1977)

3. 't Hooft, G.: Phys. Rev. Lett. 37, 8 (1976)

4. DeAlfaro, V., Fubini, S., Furlan, G.: Phys. Lett. 65B, 163 (1976)

5. Callan, C., Dashen, R., Gross, D. : Phys. Rev. 17D, 2717 (1978)

6. Glimm, J., Jaffe, A.: Phys. Rev. Lett. 40, 277 (1978); Phys. Rev. 18D, 463 (1978)

7. Glimm, J., Jaffe, A. : Phys. Lett. 73B, 167 (1978)

8. Vainberg, M.: Variational method and method of monotone operators in the theory of nonlinear equations. New York: Wiley 1973

9. Adams, R.: Sobolev spaces. New York: Academic Press 1965

10. Morrey, C.: Multiple integrals in the calculus of variations. Berlin, Heidelberg, New York: Springer 1966

Communicated by A. Jaffe

Received December 24, 1977; in revised form April 26, 1979 
